Although the first purpose of the feasibility study is to evaluate a full scale barrage, the consulting enginecrs, Sir Alexander Gibb and Partners, are also investigating the possibility of an embankment dam or dams further up the estuaries of the Kent and Leven, and the development of pumped storage reservoirs in the inter-tidal areas. At the same time, the Water Resources Board has been directing a study of the present and future demands for water in the North of England and evaluating the possible sources of supply, other than a barrage or barrages, which could meet these demands. The results of this study are expected in 1969. The Economic Study Group of the North-West Economic Planning Council is also involved in the whole scheme. It is considering the wider economic and social effects of a barrage, and its conclusions will be incorporated with the results of the engincering studies in a comprehensive report by the board to the Government in 1971 .

\section{MILITARY TRAINING}

\section{Simulating Combat}

A WELL-PLANNED but fictional tactical offensive was carried out by the staff of Ferranti Ltd in a display of its Action-Speed Tactical Trainer last Friday. The ASTT, as it is called, is a computerized system designed to train personnel in naval tactics at the Command team level, and covers seaborne, airborne and submarine units.

The system comprises twenty cubicles, each of which can be programmed to represent any one of the three types of units. The whole operation is controlled from a monitoring room with special consoles for the instructional staff and screen displays for spectators. The parameters in the game are about one hundred "tracks" which may represent missiles, echoes, decoys or any other realistic device, and ezch cubicle is equipped with controls equivalent to those in either a plane, ship or submarine. Cubicles can detect each other according to their simulated roles-by radar or sonar, for example-taking into account such effects as jamming and earth curvature. The capabilities of each cubicle can be under either monitor or programme control, so that trainees feel under combat conditions.

One of the ironies, of course, of producing a military system of this nature is that although it is clearly cut out to be of great export potential-most countries like to train their military personnel cheaply and effectively-it is likely to be subject to a variety of political embargoes. Although not in itself an offensive weapon, it can make the use of offensive weapons more effective. This argument has already prevented the company selling computational facilities for military purposes to South Africa. Orders for over $£ 2$ million have, however, been received for the ASTT from two NATO and two Commonwealth countries. It seems that the main demand will come from medium size navies.

Ferranti also took the opportunity of exhibiting a new small computer-the FM1600 B. It was originally built for military uses, but its first sale was for air traffic control, and subsequent analyses have shown it to be suitable for several civil functions. Its small size is made possible by the use of microcircuits, and Ferranti claims that it is one quarter the size and has twice the computational power of the old F1600 model.

\section{TELEVISION BROADCASTING}

\section{Sound in Vision}

The BBC gave a trial showing last week of a new method of transmitting the sound track in television programmes which it has called "sound in vision". A stimulating and elegant account of the system was presented by Mr E. R. Rout, head of the Electronics Group of the BBC Engineering Department, who managed to embellish the theory with a series of colourful field trials.

The theory underlying the new system is rery straightforward. In present usage, sound and vision signals are dispatched separately by a network of GPO cable and radio links to the various transmitting stations in the United Kingdom. The sound in vision method, however, incorporates the sound signal into that of the picture - a feature which is made possible by the fact that the vision is not transmitted as a continuous signal. The sound transmission in fact occupies the circuit for $3.8 \mu$ s of the $4.7 \mu$ s line synchronizing interval, the remainder being used for vision. (The wave form is shown in the accompanying photograph.)

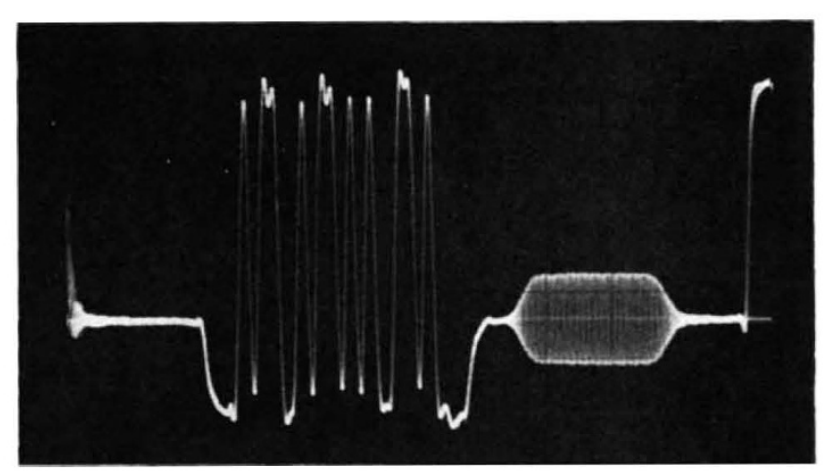

The mechanism of inserting the sound signals is by the use of pulse code modulation (PCM). Instead of inserting a continuous sound signal, the waveform is sampled at regular intervals and the resultants are coded into pulses and then added to the vision signal. A similar decoding process takes place at the receiving end, and a block diagram of the complete transmission system is shown.

It is, of course, essential that neither the sound nor the vision should suffer by this economy, and the $\mathrm{BBC}$ last week set up a series of demonstrations to allow this to be verified. The difference in quality between sending a piece of piano music to Scotland and back to London on the one hand, and round the lecture room on the other, was undetectable to an average observer, and the $\mathrm{BBC}$ claimed that the signal would stand two long British reference links with a margin of $12 \mathrm{~dB}$ with no impairment.

The present cost to the $\mathrm{BBC}$ of sound links for television is in the region of $£ 75,000$ a year, and although the cost of new equipment and its installation would initially outweigh the financial gain of introducing sound in vision, the BBC estimates that within three years the overall account should be out of the red. There is also a chance that other broadcasting authori- 